\title{
LECTOTYPES OF HAGEN SPECIES \\ BELONGING TO CERTAIN FAMILIES OF \\ TRICHOPTERA ${ }^{1}$
}

\author{
By HERBERT H. ROSS \\ Illinois Natural History Survey, Urbana
}

During the course of study of the Hagen material belonging to the primitive families of Trichoptera it appeared desirable to designate lectotypes of those species represented by a type series. This material is in the collection of the Museum of Comparative Zoology at Harvard University, Cambridge, Massachusetts. Lectotype specimens have been so labelled. In addition to these, a few unique types have been recorded here for convenience.

The species treated belong primarily to the families Philopotamidae, Psychomyiidae, Hydropsychidae and Rhyacophilidae, plus one or two in other families. The species are listed alphabetically, first by the genus in which they were originally described, then by the species names given by Hagen. In certain genera such as Macronema, Hagen included species of great taxonomic diversity; for clarity all species described by Hagen in such genera are treated here.

In addition to the labels indicated the specimens noted bear also the Hagen Collection label. The English translation of the label is given.

\section{Agapetus rudis Hagen}

1859. Verh. K.K. zool.-bot. Ges. Wien, $9: 211$.

Data in original description: Rambodde, Ceylon, Nietner coll.

Lectotype male: Ceylon, Nietner, M.C.Z. Type No. 11086.

${ }^{1}$ This study was aided by a research grant from the John Simon Guggenheim Memorial Foundation and published with a grant from the Museum of Comparative Zoology at Harvard College. 
Aphelocheira meridionalis Hagen

1864. Ann. Soc. Ent. France, ser. 4, 4:44. ô. Data in original description: Corsica (inferred from title). Lectotype male: Corsica, B. Ch., M.C.Z. Type No. 11056.

Chimarrha auriceps Hagen

1858. Verh. K.K. zool.-bot. Ges. Wien, 8:486.

Data in original description: Rambodde, Ceylon, Nietner coll.

Lectotype male: Ceylon, Nietner, M.C.Z. Type No. 11094.

\section{Chimarrha circularis Hagen}

1859. Verh. K.K. zool.-bot. Ges. Wien, $9: 210$.

Data in original description: Rambodde, Ceylon, Nietner coll.

Lectotype male: Ceylon, Nietner, M.C.Z. Type No. 11095.

\section{Chimarrha funesta Hagen}

1858. Verh. K.K. zool.-bot. Ges. Wien, 8:486.

Data in original description: Rambodde, Ceylon, Nietner coll.

Lectotype male: Ceylon, Nietner, M.C.Z. Type No. 11097.

This species belongs in the family Psychomyiidae and perhaps in the genus Pseudoneureclipsis Ulmer.

\section{Chimarrha pulchra Hagen}

1861. Synopsis Neur. N. Amer. :298.

Data in original description: Cuba (Poey, Osten Sacken). Lectotype female: Cuba, March 1858, O. Sacken, M.C.Z.

Type No. 11100.

\section{Chimarrha sepulcralis Hagen}

1858. Verh. K.K. zool.-bot. Ges. Wien, 8:486.

Data in original description: Rambodde, Ceylon, Nietner coll.

Lectotype male: Ceylon, Nietner, M.C.Z. Type No. 11096. 


\section{Hydropsyche (?) bivittata Hagen}

1861. Synopsis Neur. N. Amer. :291.

Data in original description: Panama.

Lectotype female: Panama, M.C.Z. Type No. 11019.

A member of the genus Smicridea, closely allied to fasciatella McL.

\section{Hydropsyche maderensis Hagen}

1865. Stettiner Ent. Ztg., $26: 219$.

Data in original description: Madeira.

Lectotype male: Madeira, 1852, M.C.Z. Type No. 22182.

\section{Hydropsyche maligna Hagen}

1859. Verh. K.K. zool.-bot. Ges. Wien, $9: 211$.

Data in original description: Rambodde, Ceylon, Nietner coll.

Lectotype male: Ceylon, Nietner, M.C.Z. Type No. 11023.

\section{Hydropsyche mitis Hagen}

1858. Verh. K.K. zool.-bot. Ges. Wien, 8:487.

Data in original description: Rambodde, Ceylon, Nietner coll.

Lectotype male: Ceylon, Nietner, M.C.Z. Type No. 11024.

This species belongs to the genus Tinodes (see under Polycentropus ? rufus below).

\section{Hydropsyche papilionacea Hagen}

1859. Verh. K.K. zool.-bot. Ges. Wien, $9: 211$.

Data in original description: Rambodde, Ceylon, Nietner coll.

Lectotype male: Ceylon, Nietner, M.C.Z. Type No. 11022.

Hydropsyche taprobanes Hagen

1858. Verh. K.K. zool.-bot. Ges. Wien, 8:487.

Data in original description: Rambodde, Ceylon, Nietner coll.

Lectotype male: Ceylon, Nietner, M.C.Z. Type No. 11021. 
Hydroptila cursitans Hagen

1859. Verh. K.K. zool.-bot. Ges. Wien, 9:209.

Data in original description: Rambodde, Ceylon, Nietner coll.; common.

Lectotype male: Ceylon, Nietner, M.C.Z. Type No. 11106.

Made the genotype of Plethus Hagen.

Macronema aeneum Hagen

1861. Synopsis Neur. N. Amer.: 285.

Data in original description: Mexico (Sallé).

Type female: Sallé, V.C., M.C.Z. Type No. 10992.

Probably a member of the genus Notiomyia.

Macronema annulicorne Hagen

1858. Verh. K.K. zool.-bot. Ges. Wien, 8:485.

Data in original description: Rambodde, Ceylon, Nietner coll.

Lectotype male: Ceylon, Nietner, M.C.Z. Type No. 10990.

Placed in Anisocentropus by Ulmer.

\section{Macronema ceylanicum Hagen}

1858. Verh. K.K. zool.-bot. Ges. Wien, 8:485.

Data in original description: Rambodde, Ceylon, Nietner coll.

Lectotype male: Ceylon, Nietner, M.C.Z. Type No. 11031.

The type of the genus Pseudoleptonema Mosely.

Macronema chalybeum Hagen

1861. Synopsis Neur. N. Amer. :285.

Data in original description: Cuba (Poey).

Lectotype male: Cuba, 1864, Poey, M.C.Z. Type No. 10991.

Now considered a member of the genus Phylloicus Müller.

Macronema nebulosum Hagen

1858. Verh. K.K. zool.-bot. Ges. Wien, 8:485.

Data in original description: Rambodde, Ceylon, Nietner coll.

Lectotype male: Ceylon, Nietner, M.C.Z. Type No. 11030. 
Macronema obliquum Hagen

1858. Verh. K.K. zool.-bot. Ges. Wien, 8:485.

Data in original description: Rambodde, Ceylon, Nietner coll.

Lectotype male: Ceylon, Nietner, M.C.Z. Type No. 11033.

\section{Macronema sepultum Hagen}

1859. Verh. K.K. zool.-bot. Ges. Wien, 9:209.

Data in original description: Rambodde, Ceylon, Nietner coll.

Lectotype male: Ceylon, Nietner, M.C.Z. Type No. 11032.

\section{Macronema splendidum Hagen}

1858. Verh. K.K. zool.-bot. Ges. Wien, 8:484.

Data in original description: Rambodde, Ceylon, Nietner coll.

Lectotype male: Ceylon, Nietner, M.C.Z. Type No. 11029.

\section{Macronema vitrinum Hagen}

1859. Verh. K.K. zool.-bot. Ges. Wien, $9: 209$.

Data in original description: a single male, Rambodde, Ceylon, Nietner coll.

Type male: Ceylon, Nietner, M.C.Z. Type No. 11025.

\section{Notibodia lutea Hagen}

1861. Synopsis Neur. N. Amer. :271.

Data in original description: St. Domingo.

Lectotype female: St. Domingo, Winthem, M.C.Z. Type No. 10940.

A member of the genus Helicopsyche.

\section{Philopotamus flavidus Hagen}

1864. Ann. Soc. Ent. France, ser. 4, 4:44.

Data in original description: Corsica.

Lectotype male: Corsica, B. Ch., M.C.Z. Type No. 11061. 
Philopotamus siculus Hagen

1860. Stettiner Ent. Ztg., $21: 278$.

Data in original description: 2 females from Messina, end of March.

Lectotype female: Messina, May 30, 1844, M.C.Z. Type No. 11059.

\section{Polycentropus nubigenus Hagen}

1859. Verh. K.K. zool.-bot. Ges. Wien, $9: 211$.

Data in original description: Rambodde, Ceylon, Nietner coll.

Lectotype male: Ceylon, Nietner, M.C.Z. Type No. 11034.

\section{Polycentropus ? rufus Hagen}

1859. Verh. K.K. zool.-bot. Ges. Wien, $9: 211$.

Data in original description: Rambodde, Ceylon, Nietner coll.

Lectotype male: Ceylon, Nietner, M.C.Z. Type No. 11035.

This species is a synonym of Tinodes mitis (Hagen).

\section{Rhyccophila castanea Hagen}

1858. Verh. K.K. zool.-bot. Ges. Wien, 8:487.

Data in original description: Rambodde, Ceylon, Nietner coll.

Lectotype male: Ceylon, Nietner, M.C.Z. Type No. 11079.

Rhyacophila fasciata Hagen

1859. Stettiner Ent. Ztg., $20: 153$.

Data in original description: A single male from Elberfeld. Type male: M.C.Z. Type No. 11067. Bears a label which says " 57 ", but no other data.

\section{Rhyacophila paupera Hagen}

1859. Stettiner Ent. Ztg., $20: 153$.

Data in original description: Prussia, Schlesien, on the Rhein (?), Archangel.

Lectotype female: M.C.Z. Type 11066, and a label bearing a word which might be "Schlesien". 

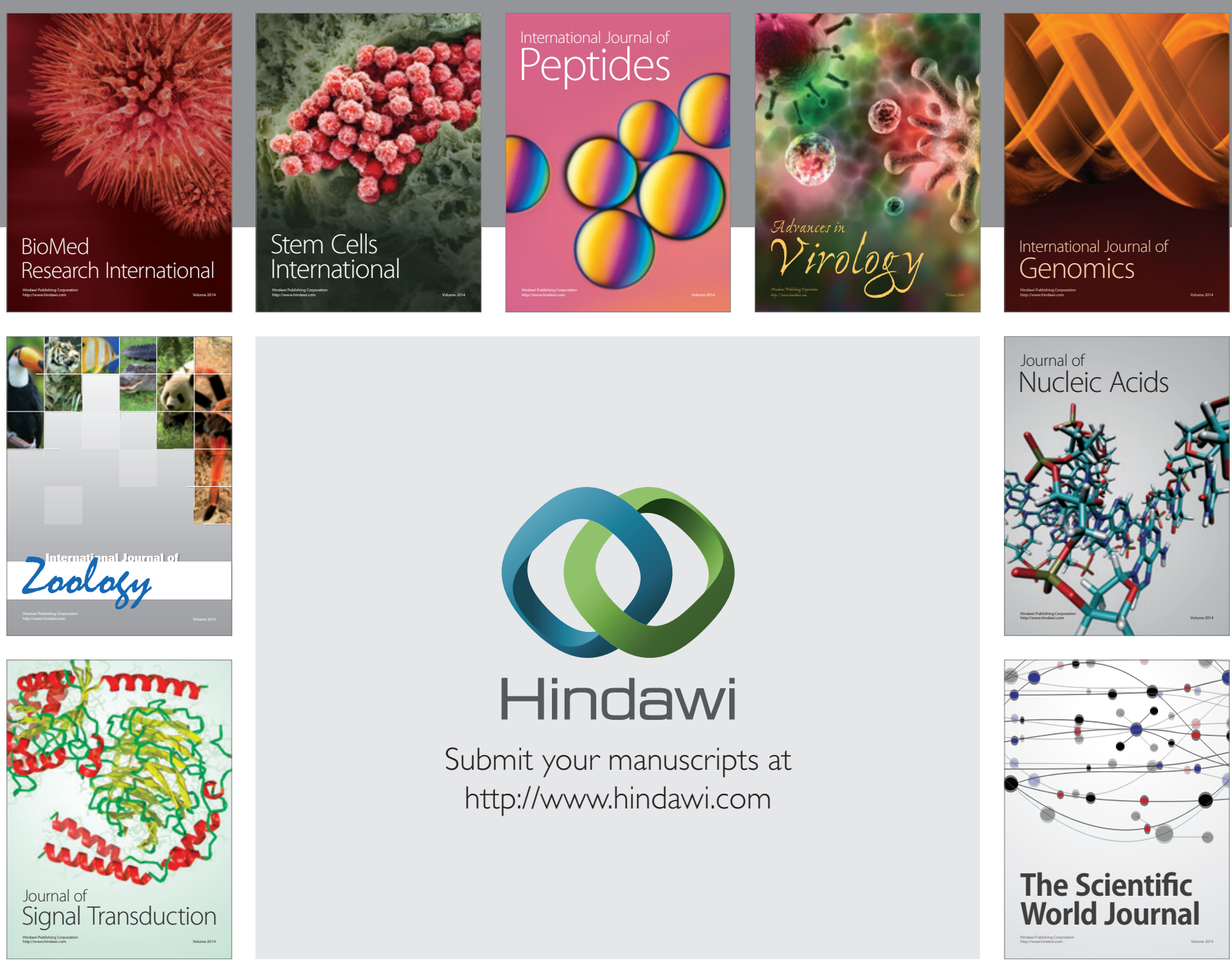

Submit your manuscripts at

http://www.hindawi.com
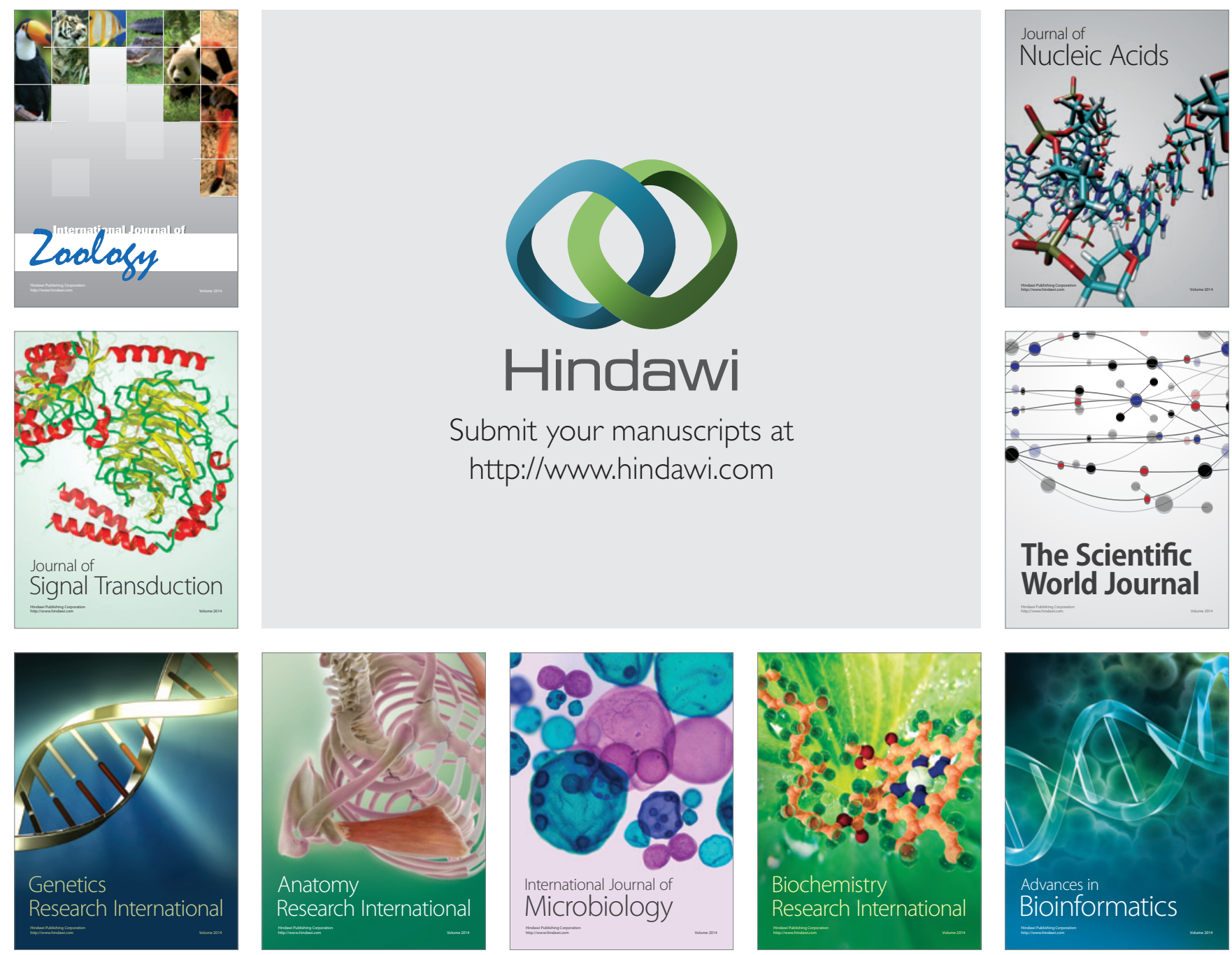

The Scientific World Journal
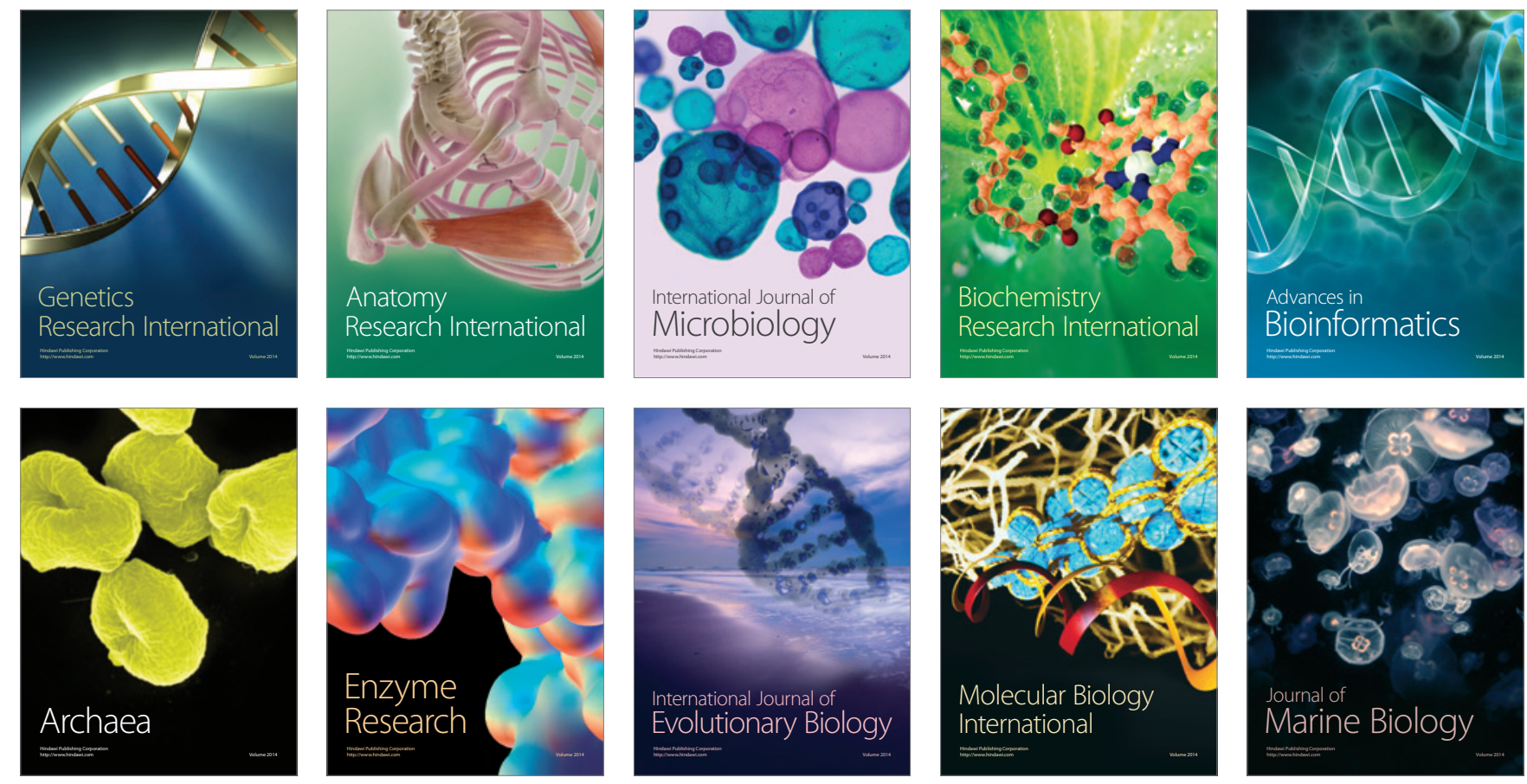